\title{
ORIGINAL
}

\section{MEDICAMENTOS GENÉRICOS, PERCEPCIÓN DE LOS MÉDICOS. CALI-COLOMBIA}

Programa de Medicina, Universidad Libre, Cali-Colombia

${ }^{1}$ Médico-Farmacólogo, PhD en Ciencias de la Educación. Investigador del Instituto de Investigaciones Biomédicas de la Universidad Libre y Profesor Titular de Farmacología,

Universidad Santiago de Cali. Colombia.

2 Estudiante, Programa de Medicina, Universidad Libre, Seccional de Cali, Colombia.
Palabras clave: prescripción de medicamentos, medicamentos genéricos, medicamentos innovadores, medicamentos fraudulentos

Keywords: prescription of drugs, generic drugs, innovative medicines, fraudulent drugs.

Correspondencia a: Email: catorse@yahoo.com

Recibido para publicación:

Aceptado para publicación: 20 de mayo de 2018

Citar como:

Rev Cient Cienc Med

2018;21(1):40-49

\section{GENERIC MEDICATIONS, PERCEPTION OF THE DOCTORS. CALI, COLOMBIA}

Camilo Torres Serna ${ }^{1}$, Juan Camilo Ángel Medina ${ }^{2}$, Helen Viviana Klinger Torres ${ }^{b}$, Vanessa Márquez Flórez ${ }^{b}$, Jean Marcos Micolta Bejarano ${ }^{b}$, Jhon Jairo Sánchez Suescún ${ }^{b}$.

\section{RESUMEN}

Es frecuente escuchar la desconfianza médica de la efectividad de los medicamentos genéricos. El objetivo de este estudio es cuantificar la aceptación de médicos vinculados al Sistema General de Seguridad Social en Salud de Colombia a la prescripción de medicamentos genéricos.

Se realizó un estudio de tipo descriptivo de corte transversal. Se entrevistaron 80 médicos en ejercicio clínico, todos médicos que realizaban consulta clínica en los sitios de práctica de una Facultad de Medicina de Cali-Colombia.

Los resultados muestran que los médicos consultados tienen claro el concepto de medicamento genérico y el $89,6 \%$ de ellos, consideran el producto genérico esencialmente similar al medicamento original de referencia, pero al mismo tiempo tienden a pensar que los productos genéricos no cumplen con el efecto terapéutico deseado. Se destaca también que el $74,4 \%$ de los médicos considera que los pacientes valoran poco el uso de productos genéricos.

\section{ABSTRACT}

It is common to hear medical mistrust of the effectiveness of generic drugs. The objective of this study is to quantify the acceptance of physicians linked to the General System of Social Security in Health of Colombia to the prescription of generic drugs.

Was performed a cross-sectional, descriptive study. Eighty doctors in clinical practice were interviewed, all doctors who made clinical consultation in the practice sites of a Medical School of Cali-Colombia.

The results show that the physicians consulted are clear about the concept of generic medicine and $89.6 \%$ of them consider the generic product essentially similar to the original reference medicine, but at the same time, they tend to think that generic products do not comply with the desired therapeutic effect. It is also stand out that $\mathbf{7 4 , 4 \%}$ of doctors consider that patients value the use of generic products little.

\section{INTRODUCCIÓN}

a gestión y administración del uso de mediLcamentos constituye un factor determinante del equilibrio financiero de una organización de salud. Frecuentemente las organizaciones deben establecer y revisar procesos de licitación, compra, distribución y suministro adecuado de medicamentos a sus usuarios y en esta área se presentan grandes posibilidades de investigación administrativa.

En todo el mundo existe gran controversia alrededor del uso de medicamentos innovadores y de sus similares llamados medicamentos genéricos. Las entidades de salud prefieren comprar productos genéricos la mayoría de las veces por ser más económicos pero muchos médicos y pacientes plantean ineficacia de esos productos.

Se conoce como medicamento genérico aquel que es técnicamente igual al primer medicamento investigado con esa composición quími$\mathrm{ca}$, denominado medicamento innovador. Un laboratorio investigador desarrolla una molécula innovadora con usos terapéuticos e inicia su comercialización con una marca registrada, esta nueva molécula tiene reserva o patente de propiedad intelectual durante veinte años, posteriormente otros laboratorios empiezan a fabricar la misma molécula y a comercializarla con la Denominación Común Internacional (DCl) o con otras marcas secundarias. Estos pro- 
ductos, con la misma composición, despectivamente llamados copias, son los que deben denominarse productos genéricos, en algunos países se conocen como productos similares o como genéricos intercambiables (México), la Organización mundial de la Salud sugiere que se les denomine medicamentos de origen multifuente .

El objetivo de este trabajo fue el de identificar el concepto y la opinión que, sobre el medicamento genérico, tiene el médico que trabaja en el Sistema General de Seguridad Social en Salud (SGSSS) de Colombia, sistema que atiende más del $90 \%$ de la población colombiana. Se espera que la información obtenida permita orientar campañas de capacitación y de información acerca de la bioequivalencia de medicamentos y sustentar con cifras, la importancia de lograr una racionalización del uso de los medicamentos por parte de médicos, farmacéuticos, y pacientes; además contribuir al control del uso innecesario de sustancias farmacológicas más costosas.

\section{MATERIALES Y MÉTODOS}

Se realizó un estudio de tipo descriptivo transversal. Para recoger la información se utilizó el cuestionario mixto reconocido por la sigla PAMPEFG-01 (Percepción y Actuación de Médicos en la Prescripción de Especialidades Farmacéuticas Genéricas), instrumento validado y utilizado por García AJ., et al. y adaptado para este estudio.

El universo fueron todos los médicos, generales y especialistas, que cumplían el requisito de inclusión, de trabajar en servicios de atención médica directa (servicios de emergencia y de consulta externa), en los sitios de práctica clínica de la Facultad de Medicina de la Universidad Libre en Cali-Colombia, se excluyeron los médicos que no realizaban ningún tipo de consulta médica, como aquellos que trabajaban en actividades unicamente administrativas. Se encontró que en los 7 sitios de práctica, de nivel 1, 2 y 3 de complejidad médica, cumplían el requisito de inclusión un total de 245 médicos generales y especialistas en diferentes turnos y bajo diferentes modalidades de contratación (tiempo completo, medio tiempo o prestación de servicios por evento), aplicando un software calculador de muestra (Rotator Survey ${ }^{\circledR}$ ), se obtuvo que la muestra mínima debería ser de 70 médicos, los cuales fueron seleccionados de manera probabilística el día escogido para recoger la información. Cada médico fue entrevistado independientemente, garantizándole el tiempo necesario para responder el cuestionario y la debida confidencialidad. Esta investigación forma parte de un macroproyecto acerca del uso social del medicamento que realiza el grupo Gestión en Salud del programa de Gerencia en Salud y el Instituto de Investigaciones Biomédicas de la Universidad Libre de Cali, Colombia. Los médicos participantes en el estudio, tuvieron información de la finalidad de la investigación y fueron invitados a participar voluntariamente y mediante consentimiento informado. Según las normas y estándares del Ministerio de Salud de Colombia, esta investigación fue consi-

derada sin riesgo porque no se realizó ninguna intervención o modificación intencionada de las variables biológicas, fisiológicas, psicológicas o sociales de los sujetos participantes en el estudio, también se realizó un cuestionario en el que no se identificó al médico participante, no se solicitaron datos personales y tampoco se involucraron aspectos sensitivos de su conducta. El comité de evaluación ética y científica para la investigación en seres humanos de la Universidad Libre de Cali aprobó la realización de este estudio mediante acta 05 de 2016.

\section{RESULTADOS}

Se entrevistaron 80 médicos, 59 hombres y 21 mujeres, en ejercicio clínico (consulta externa o emergencias), 53 de ellos eran mayores de 31 años (tabla 1), 41 eran médicos generales y 39 tenían alguna especialidad clínica. La mitad de los médicos tenían más de 10 años de experiencia profesional y solo 3 tenían menos de un año de experiencia profesional. (tabla 2)

Las preguntas del formulario fueron analizadas en dos direcciones, las preguntas relacionadas con el conocimiento que tenían los médicos sobre el concepto de medicamento genérico (tabla 3), y las preguntas relacionadas con la confianza del médico en el medicamento genérico (tabla 4). Las respuestas muestran un buen conocimiento del concepto pues el $69 \%$ reconoce la diferencia entre medicamento esencial definido según la OMS, como aquellos medicamentos que satisfacen las necesidades prioritarias de salud en la población, y medicamento genérico y el $73 \%$ considera que no se debe confundir un medicamento genérico con un producto ilegal o popularmente llamado producto

\begin{tabular}{|c|c|c|c|}
\hline Edades (años) & Hombres & Mujeres & Total \\
\hline 30 o menos & 14 & 13 & 27 \\
\hline $31-40$ & 8 & 5 & 13 \\
\hline $41-50$ & 12 & 3 & 15 \\
\hline $51-60$ & 22 & 0 & 22 \\
\hline Menos de 61 & 3 & 0 & 3 \\
\hline Total & 59 & 21 & 80 \\
\hline
\end{tabular}

Tabla 2 Años de experiencia profesional de los médicos participantes en el estudio de percepción acerca de medicamentos genéricos.

\begin{tabular}{llll}
\hline Años de experiencia & Hombres & Mujeres & Total \\
\hline Menos de 1 & 2 & 1 & 3 \\
\hline $1-5$ & 13 & 13 & 26 \\
\hline $5-10$ & 5 & 5 & 10 \\
\hline Mas de 10 & 39 & 2 & 41 \\
\hline Total & 59 & 21 & 80 \\
\hline
\end{tabular}


Tabla 3

Conocimiento del concepto de medicamento genérico por parte de los médicos participantes en la encuesta.

\begin{tabular}{clcc}
\hline \multirow{2}{*}{ Pregunta } & \multicolumn{1}{c}{ Conocimiento acerca del medicamento genérico } & $\begin{array}{c}\text { En desacuerdo con lo } \\
\text { afirmado (\%) }\end{array}$ & $\begin{array}{l}\text { De acuerdo con lo } \\
\text { afirmado (\%) }\end{array}$ \\
\hline 6 & Medicamentos esenciales y medicamentos genéricos son la misma cosa & 69,33 & 21,33 \\
\hline 7 & $\begin{array}{l}\text { Los medicamentos que suministra el sistema de salud solo pueden ser genéri- } \\
\text { cos }\end{array}$ & 68,83 & 27,27 \\
\hline 12 & $\begin{array}{l}\text { Los genéricos deben ser esencialmente similares al medicamento original de } \\
\text { referencia. }\end{array}$ & 9,09 & 89,61 \\
\hline 14 & $\begin{array}{l}\text { La prescripción de genéricos disminuye el gasto farmacéutico al Sistema de } \\
\text { Salud }\end{array}$ & 19,23 & 73,08 \\
\hline 15 & $\begin{array}{l}\text { Los genéricos deben usarse en sustitución de los medicamentos patentados } \\
\text { debido a su bajo costo }\end{array}$ & 53,95 & 32,89 \\
\hline 17 & $\begin{array}{l}\text { En mi institución de Pr estación de Servicios (IPS) se prioriza medicamentos } \\
\text { comerciales }\end{array}$ & 16,88 & 75,32 \\
\hline 19 & $\begin{array}{l}\text { Los médicos suelen obtener regalos o premios por receta medicamentos co- } \\
\text { merciales }\end{array}$ & 43,59 & 51,28 \\
\hline 21 & $\begin{array}{l}\text { La mayoría de genéricos que existen en el mercado son ilegales (productos } \\
\text { piratas) }\end{array}$ & 73,69 & 15,79 \\
\hline 22 & $\begin{array}{l}\text { El uso de los genéricos limita la creación e investigación de nuevos medicamen- } \\
\text { tos }\end{array}$ & 69,23 & 21,79 \\
\hline
\end{tabular}

\section{Tabla 4}

Confianza en los medicamentos genéricos de los médicos participantes en la encuesta.

\begin{tabular}{llll}
\hline Pregunta & Confianza en el medicamento genérico & $\begin{array}{l}\text { En desacuerdo con lo } \\
\text { afirmado (\%) }\end{array}$ & $\begin{array}{l}\text { De acuerdo con } \\
\text { lo afirmado (\%) }\end{array}$ \\
\hline 8 & $\begin{array}{l}\text { Medicamentos tienen la misma bioequivalencia y biodisponibilidad que los medicamentos no } \\
\text { genéricos }\end{array}$ & 52,63 & 35,53 \\
\hline 9 & Los genéricos consiguen el mismo efecto clínico que los medicamentos no genéricos & 48,05 & 37,66 \\
\hline 10 & $\begin{array}{l}\text { Generalmente los medicamentos genéricos no cumplen con el efecto terapéutico (tratamiento } \\
\text { fallido). }\end{array}$ & 62,34 & 22,08 \\
\hline 11 & El uso de genéricos aumenta el riesgo de efecto indeseables & 67,1 & 23,7 \\
\hline 13 & Los genéricos ofrecen las mismas garantías de calidad del preparado por una marca comercial. & 51,3 & 35,9 \\
\hline 16 & Normalmente receto (o me recetan) medicamentos genéricos & 19,2 & 71,8 \\
\hline 18 & El uso de genéricos disminuye la capacidad de elección del médico para recetar medicamen- & 45,5 & 49,4 \\
\hline 20 & tos. & 37,2 & \\
\hline 23 & Se duda la calidad de los laboratorios que fabrican genéricos. & 16,7 & 44,9 \\
\hline
\end{tabular}

pirata. El $89,6 \%$ considera que los genéricos deben ser iguales a los originales y el $69 \%$ no considera que el uso de medicamentos genéricos limite la investigación farmacológica. Con respecto a las preguntas relacionadas con la confianza que el médico tiene del medicamento genérico (tabla 4), se puede destacar que el $71,8 \%$ usa medicamentos genéricos, el $62,3 \%$ no encuentra fallas terapéuticas y el $67,1 \%$ no considera que aumenten los efectos indeseables. Un resultado crítico es que los médicos entrevistados consideran que los pacientes le dan poco valor al uso de genéricos.

En la tabla 4, se resumen los porcentajes de respuesta a las preguntas relacionadas con el conocimiento del concepto de medicamento genérico que tenían los médicos entrevistados, se comparan las respuestas relacionadas con el acuerdo o no a la premisa planteada y se descartan los resultados marcados como indecisos.

\section{DISCUSIÓN}

Es importante, para los actores de un sistema de salud tener conceptos claros sobre los mitos y las realidades del uso de los medicamentos genéricos. El término genérico no se refiere únicamente al producto farmacéutico que se comercializa con el nombre del principio activo como muchas personas creen. El término producto genérico se refiere al medicamento que no es el producto originalmente desarrollado por una empresa farmacéutico sino el producto idéntico fabricado por otra empresa farmacéutica, existe mucha evidencia de la bioequivalencia química, farmacéutica y clínica de los productos originales con los productos genéricos.

Los resultados de este estudio muestran que lo médicos consultados tienen claro el concepto de medicamento genérico (tabla 3), y el $89,6 \%$ de ellos, consideran que el producto genérico debe ser esencialmente similar al medicamento original de re- 
ferencia, al mismo tiempo tienden a pensar $(62,3 \%)$ que los productos genéricos cumplen con el efecto terapéutico deseado. Se destaca también que el $74,4 \%$ de los médicos considera que los pacientes valoran poco el uso de productos genéricos.

Pese a que los medicamentos genéricos tienen la misma efectividad que los de marca y son más baratos, los resultados de este estudio y muchos otros muestran que existen porcentajes importantes de médicos (cerca del $40 \%$ en este estudio), que tienen preferencia por los productos originales y piensan que sus pacientes también los prefieren. El Dr. Óscar Andia, Director del Observatorio del Medicamento de la Federación Médica Colombiana (Observamed), plantea que en Colombia hay un imaginario, tan arraigado como falso, de que los genéricos son de peor calidad citado por Silva S. Una buena muestra de eso es que, inclusive, los médicos prefieran recomendar esa opción.

Según el Instituto Colombiano de Vigilancia de Medicamentos y Alimentos (Invima), los genéricos en Colombia se fabrican con altos estándares de calidad y cuestan menos porque no incluyen los costos de investigación y desarrollo o los costos de las patentes, además sus fabricantes gastan menos en publicidad y promoción.

Un meta síntesis de estudios que comparan el uso de genéricos y no genéricos (productos innovadores u originales), en situaciones médicas claves se mencionan a continuación.

En la Universidad del Valle en Cali Colombia, se comparó la biodisponibilidad de dos productos de alto involucramiento, el inmunosupresor (ciclosporina original), con un producto genérico y la de una heparina de bajo peso molecular (enoxaparina original), con uno de sus genéricos. En ambos casos, no se encontraron diferencias significativas en los parámetros farmacocinéticos medidos, ni en área bajo la curva $(A B C)$, ni en concentración máxima (Cmax), ni en el tiempo para lograr la concentración máxima (Tmax).

Kesselheim, Misono, et al. realizaron una búsqueda sistemática de publicaciones en MEDLINE, EMBASE y en el International Pharmaceutical Abstracts, encontraron 47 artículos sobre análisis detallados que cubrían nueve de las diferentes subclases de fármacos cardiovasculares, de los que el $81 \%$ eran ensayos clínicos aleatorios. La equivalencia clínica se probó en los siete ensayos sobre bloqueadores beta encontrados, en 10 de 11 revisados sobre diuréticos, en cinco de los siete ensayos sobre antagonistas del calcio, en los tres que investigaron antiplaquetarios, en los dos ensayos sobre estatinas, en uno realizado sobre inhibidores de la ECA y en otro sobre bloqueadores adrenérgicos alfa. También analizaron 53 editoriales y comentarios, en ellos un $53 \%$ expresaban un punto de vista negativo sobre los genéricos en comparación con un $28 \%$ que alentaba la sustitución de los medicamentos innovadores por sus genéricos.

La sustitución por genéricos de los fármacos antihipertensivos no conduce a un menor seguimiento o aumenta las hospitalizaciones relacionadas con enfermedades cardiovasculares, en comparación con la terapia de marca. Cuando está disponible un antihipertensivo equivalente genérico, se debe considerar la sustitución del producto original por un genérico, para lograr beneficios económicos y asegurar el tratamiento crónico .

En otras especialidades de la medicina, se encuentran estudios con resultados parecidos, Vlahiotis y Devine, en pacientes con episodio depresivo mayor, no observaron una mayor tasas de abandono del tratamiento entre aquellos que empiezan el tratamiento farmacológico con un genérico y los que comienzan con un medicamento de marca original.

Una de las mayores controversias que se encuentran en la literatura, es la sustitución de medicamentos originales por genéricos, en epilépticos. Polard, et al. estudiaron la asociación entre la sustitución genérica y la pérdida del control de las crisis, analizaron un total de 8379 pacientes y los análisis de sensibilidad arrojaron resultados similares. Los autores concluyeron que la sustitución de anticonvulsivos originales por genéricos no se asoció con un mayor riesgo elevado de convulsiones.

Pereira, et al. compararon el efecto anticoagulante de warfarinas orales a través de su efecto sobre el International Normalized Ratio (INR) y no encontraron diferencias entre productos originales y genéricos que obligaran a ajustes de dosis. Tampoco encontraron diferencia en la variación de INR basada en la formulación de warfarina ( $p>0,69)$, ni se encontró interacción entre el paciente y la warfarina $(p>0,81)$. Los resultados INR no fueron influenciados por si los pacientes fueron mantenidos con el producto original solamente (grupo de control), o intercambiados entre el producto original y warfarinas genéricas ( $p=0.98)$. La conclusión de estos estudios es que los pacientes pueden cambiar con seguridad y eficacia entre la warfarina original y la genérica.

Como lo plantean Duerden y Hughes, a pesar de que la sustitución de productos innovadores por sus genéricos, puede afectar a algunos pacientes individuales, sobre todo en la reducción de la adherencia y el aumento potencial de errores de medicación, podría ser un precio que vale la pena pagar, dada la oportunidad de ahorro asociada con el uso de medicamentos que clínicamente no son mejores que las alternativas más baratas.

Las aseguradoras y los creadores de políticas farmacéuticas fomentan el uso de medicamentos genéricos para reducir costos, pero los genéricos siguen infrautilizados, médicos y pacientes están de acuerdo en que los medicamentos genéricos son menos costosos y son igual de seguros.

Otro punto a destacar, en el presente estudio, es que el $74,4 \%$ de los médicos considera que los pacientes valoran poco el genérico. Este hallazgo coincide con otros estudios, por ejemplo Shrank encontró que aunque el 56\% de los estadounidenses piensan que se deberían usar más genéricos, solo el $37.6 \%$ prefiere tomar genéricos. Hay varias prácticas que las autoridades de salud, en todo el mundo, han intentado combatir para cambiar estas creencias. Una de ellas según la OMS, que la industria farmacéutica ha hecho grandes esfuerzos para desacreditar los medicamentos genéricos. "Lo usual es que se estigmatice a los genéricos mostrándolos como alternativa 'para pobres' y de mala calidad", los estados deben combatir con igual fortaleza estas prácticas.

Puede concluirse que muchos estudios, consideran que los medicamentos genéricos poseen la misma eficacia y seguridad que los medicamentos de marca y su introducción y difusión en el mercado supone un ahorro en el gasto farmacéutico que los convierte en una medida necesaria para el sostenimiento del Sistema de Salud.

Es importante tener en cuenta que en el mercado también existen productos falsificados o ilegales, los cuales no fueron objeto de estudio del presente trabajo, estos son productos que 
no cumplen, bajo ninguna circunstancia, las condiciones para ser considerados un genérico, por eso se debe tener identificado el origen del medicamento genérico para poder asegurar su bioequivalencia.

Para reducir la susceptibilidad tanto de los pacientes como de médicos, sobre el uso de medicamentos genéricos debe emplear tiempo para informar a los pacientes y formación para los médicos prescriptores en el uso racional del medicamento. Es fundamental que los médicos y farmacéuticos comprendan correctamente la sustitución de medicamentos originales por genéricos aprobados por los entes regulatorios de cada país y proporcionen la información correcta a los pacientes.

Resultados de este y otros estudios parecen demostrar que se necesitan más esfuerzos para que los colombianos confíen en estas medicinas que pueden ahorrarle al sistema de salud una buena cantidad de recursos.

\section{Agradecimientos:}

Los autores agradecen al Instituto de Investigaciones Biomédicas, Grupo de investigación de la Universidad Libre de Cali (Categoría A de Colciencias) el apoyo logístico suministrado para la realización de este trabajo.

\section{Conflicto de interés:}

Todos los autores tuvieron participación activa en la elaboración del proyecto, la recolección de la información, el análisis y la redacción del artículo y declaran no tener conflicto de interés en la realización de este trabajo. No tienen vínculos ni directos, ni indirectos, con la industria farmacéutica.

\section{REFERENCIAS}

1.McLachlan AJ. Frequently asked questions about generic medicines. Aust Prescr [Internet].2007 (citado 5 de octubre de 2017); 41-3. Disponible en: https://cdn0. scrvt.com/08ab3606b0b7a8ea53fd0b40b1c44f86/fc20fd821a02cf80/8d2289edb5e3/ef59843b3f060818930abb15d5ff4853aaeed461243034ea30c5d77ac475.pdf

2.Velásquez G. Pascale P; Organización Mundial de la Salud. Globalización y acceso a los medicamentos: Perspectivas sobre el acuerdo Adpic/Omc. 2 ed.; 2000. Disponible en: http://apps.who.int/medicinedocs/pdf/whozip47s/whozip47s.pdf

3.Consumers' Association. Generic medicines: can quality be assured?. Drug Ther Bull. 1997; 35(2): 9-11.

4.Gaither C, Kirking D, Ascione F, Welage L. Consumers' views on generic medications. J Am Pharm Assoc[Internet].2001(citado 22 de marzo de 2017); 41: 729-36. Disponible en: https://www.sciencedirect.com/science/article/pii/S1086580216313080?via\%3Dihub

5.World Health Organization, Action Programme on Essential Drugs. Global comparative pharmaceutical expenditures. Geneva: WHO; 1997.

6.Al-Jazairi AS, Bhareth S, Eqtefan IS, Al-Suwayeh SA. Brand and generic medications: are they interchangeable?. Ann Saudi Med.2008; 28(1):33-41

7.OMS. Medicamentos Esenciales. Organización Mundial de la Salud, Ginebra, 2007 8.Espinosa $\mathrm{O}$, Pinzón D. Análisis cuantitativo de la estacionalidad del gasto en salud en Colombia. Rev Monitor Estratégico [Internet].2018 (citado 10 de febrero de 2018); 13: 20. Disponible en: https://revistas.udem.edu.co/index.php/economico/article/viewFile/2365/2003

9.García AJ, Martosa F, Leivab F, Sánchez de la Cuesta F. Genéricos: ¿buenos o malos? Conocimientos y actitudes de los médicos ante los medicamentos genéricos. Gac Sanit [Interenet]. 2003;17(2):144-9. Disponible en: http://www.scielo.br/pdf/ gs/v17n2/breve.pdf

10.Isaza S. Siete verdades sobre medicamentos genéricos en Colombia. 1er. foro nacional "La verdad sobre los medicamentos genéricos". Bogotá, 2009 [Citado 15 de noviembre 2017]. Disponible en: http://colegiomedico.cundibogota.googlepages.com/ FMC_PonenciaSenado21abr09FINAL.doc

11.Bolaños VH, D'Alessio R, Fefer E, de Joncheere K, Holzhauser de Uzcátegui E. Armonización de la reglamentación farmacéutica en América Latina. Washington, DC: Organización Panamericana de la Salud; 1996.

12.Davit BM, Nwakama PE, Buehler GJ, Conner DP, Haidar SH, Patel DT. et al. Comparing generic and innovator drugs: a review of 12 years of bioequivalence data from the United States Food and Drug Administration. Ann Pharmacother. 2009;43(10):1583-97.

13.Meredith P. Bioequivalence and other unresolved issues in generic drug substitution. Clin Ther.2003;25(11):2875-90.

14.Silva S. Los colombianos no saben comprar acetaminofén. [Citado 12 de mayo 2018]. Disponible en: https://www.elespectador.com/noticias/salud/colombianos-no-saben-comprar-acetaminofen-articulo-754430

15.Grupo de comunicaciones del Invima. Los medicamentos genéricos y los originales de marca tienen los mismos estándares de calidad. Instituto Nacional de Vigilancia de Medicamentos y Alimentos de Colombia. noviembre 2016. Disponible en: https://www. invima.gov.co/los-medicamentos-gen\%C3\%A9ricos-y-los-originales-de-marca-tienen-los-mismos-est\%C3\%Alndares-de-calidad.html

16.Torres C, Rubiano J, Caicedo LA, Carvajal R. Bioequivalencia de dos presentaciones comerciales de ciclosporina oral. Anuario de Investigaciones 2000, Escuela de Medicina, U. del Valle. Cali, 2000.

17.Torres C, Vargas B, Noguera A, Rodríguez M, Vargas M, García O, et al. Estudio de biodisponibilidad comparativa y bioequivalencia de dos presentaciones de enoxaparina sódica. [Internet]. 2006 [Citado 15 de noviembre 2017]. Disponible en: http://www.susmedicos.com/0_Articulos_General/Art_enoxaparina-sodica.html

18. Kesselheim AS, Misono AS, Lee JL, Stedman MR, Brookhart MA, Choudhry NK, et al. Clinical equivalence of generic and brand-name drugs used in cardiovascular disease: a systematic review and meta-analysis. JAMA. 2008;300:2514-26.

19.Van Wijk LG, Klungel OH, Heerdink ER, de Boer A. Generic substitution of antihypertensive drugs: does it affect adherence?. Ann Pharmacotherapy.2006;40:15-20. 20.Vlahiotis A, Devine S, Eichholz J, Kautzner A. Discontinuation Rates and Health Care Costs in Adult Patients Starting generic Versus Brand SSRI or SNRI Antidepressants in Commercial Helth Plans. J Managed Care Pharmacy. 2011;17:65-87

21.Polard E, Nowak A, Happe A, Biraben E. Brand-name to generic substitution of antiepileptic Drugs (AED) does not lead to seizure-related hospitalization: a Population-based case-crossover study. Clinical Therapeutics. 2015;37:e114.

22.Pereira JA, Holbrook AM, Dolovich L, Goldsmith C, Thabane L, Douketis JD, et al. Are brand-name and generic warfarin interchangeable?. Ann Pharmacother.2005;39(7-8):1188-1193

23.Duerden MG, Hughes DA. Generic and therapeutic substitutions in the UK: are they a good thing?. British J Clin Pharm. 2010;70:335-41.

24.Meredith PA. Generic drugs: therapeutic equivalence. Drug Saf .1996;15(4):233242

25.Calvert RT. Bioequivalence and generic prescribing. J Pharm Pharmacol.1996;48(1):9-10

26.Shrank WH, Cox ER, Fischer MA, Mehta J, Choudhry NK. Patients' perceptions of generic medications. Health Aff (Millwood). 2009;28(2):546-56.. PubMed PMID: 19276015; PubMed Central PMCID: PMC2748784. Disponible en: doi: 10.1377/hlthaff.28.2.546

27.OMS. Comisión sobre determinantes sociales de la salud. Informe final. WHO/ OMS [Internet]. 2009 [Citado 20 de enero 2018]. Disponible en: www.who.int/social_ determinants/thecommission/finalreport/es

28.Verbeeck RK, Kanfer I, Walker RB. Generic substitution: the use of medicinal products containing different salts and implications for safety and efficacy. Eur $\mathrm{J}$ Pharm Sci. 2006; 28 (1-2): 1-3

29. Meyer MC. Generic drug product equivalence. Am J Manag Care. 1998;4(8):118392.

30.Bhosle, Deepak Sayyed, Asif; Bhagat, Abhijeet; Shaikh, Huzaif; Sheikh, Alimuddin; Bhopale, Vasundhara; Quazi, Zubair. Comparison of Generic and Branded Drugs on Cost Effective and Cost Benefit Analysis. Ann Int medical and Dental Research.2016;3(1):35-7. Disponible en: doi:10.21276/aimdr.2017.3.1.PC1

31.Goldman DP, Joyce GF, Zheng Y. Prescription drug cost sharing. JAMA. 2007;298(1):61-9.

32.OMS. Medicamentos falsificados-Pautas para la formulación de medidas para combatir los medicamentos falsificados. Geneva, World Health Organization.1999. Disponible en: https://www.paho.org/hq/dmdocuments/2013/GCFM-Antecedentes-esp. $p d f$

33.Murphy JE. Generic substitution and optimal patient care. Arch Intern Med.1999;159(5):429-33

34.Kobayashi E, Karigome H, Sakurada T, Satoh N, Ueda S. Patients' attitudes towards generic drug substitution in Japan. Health Policy. 2011;99:60-5. 